disappear in the course of a few hours (sometimes with a marked collapse); the frequency of the pulse may become diminished by more than one half, and the temperature may go down $10^{\circ}$ Fahr. in less than ten hours. The skin becomes bathed in perspiration; the pain in the head and limbs usually disappears, the patient seems to have entered convalescence, when after about eight clays, in the great majority of cases, more or less, but not quite suddenly, a second attack commences, with symptoms analogous to the first, but generally less severe, usually lasting from three to five days, terminating as abruptly as the former, and being followed by perfect convalescence except in rare cases, in whom a milder and shorter third (and possibly even fourth) attack may occur. The first signs of the attack usually manifest themselves towards the evening, and those of the crisis after midnight. During the convalescence there is at first a certain degree of anæmia, reminding one of the effects of ague, and the force and frequency of the pulse and the temperature of the body are less than normal.

$$
\text { (To be concluded.) }
$$

\section{ON \\ CHROMATOSCOPY AS A MEANS OF DTA- GNOSIS IN OPHTHALMIC MEDICINE.}

\section{BY J. R. WOLFE, M.D., F.R.C.S. EDIN.,} SURGEON TO THE GLASGOW OPHTHALMIC INSTITUTIOA.

When Professor Harley published, in The LanceT, * his most interesting auto-clinical observations on his attack of glaucoma, retinitis, \&c., and the subsequent process of restoration of his sight, I was desirous of making some remarks on the phenomena of perverted colour-vision. I was convinced that this was a rich field of diagnosis in ophthalmic medicine which deserved cultivation, for it might serve us as a means of distinguishing retinal affections in cases where the ophthalmoscope throws no light upon the pathological changes. I refrained, however, from doing so, being aware that my friend Dr. Galezowski, of Paris, was at that time engaged in preparing for the press the results of his very extensive investigations on that subject. His work has now appeared, and having tested his results in my clinique for the last few months, I propose to submit in this paper a brief analysis of that excellent work, in the hope that it may stimulate further inquiry.

Ever since the celebrated Dalton published his own case of congenital colour-blindness, $t$ this subject has occupied the attention of observers in all its bearings, whether physical, physiological, or pathological. I need only mention the names of Thomas Young, Brewster, Herschel, Wilson, Goethe, Helmholtz, Szokalski, and a host of other philosophers. By their labours a large number of facts have been gradually accumulated; and what now remains to be done is to systematise these facts, so as to render them available for diagnosis. This Galezowski has attempted in his work, and if he has not actually established a perfectly well defined diagnostic means, he has certainly opened the way for other investigators, in furnishing them with valuable landmarks, and in giving chromatic tables to facilitate further researches. It is, perhaps, scarcely necessary to refer, as the author does, to the fundamental physical laws of colour; I shall therefore take for granted that these are too well known to require recapitulation, and shall proceed at once to detail his views of their physiological bearing.

The nervous elements of the retina form, according to Robin, eight layers, represented in Fig. 1.

The respective functions of these different elements are, as yet, imperfectly known; but it seems probable, and Kölliker assumes it as well established, that the rods and cones are the special elements for the reception of the impressions of light.t This seems to be borne out by the circumstance that the acuteness of vision is in direct proportion to the number and distribution of these elements, as

*Vol. i. 1868, p. 158.
+ Manchester Philosophical Transactions, 1792.

† Kölliker's Microscopic Anatomy, p. 569. shown, for example, in the macula, which is composed of closely grouped cones, and which is the spot where vision is most distinct.

FIG. 1.

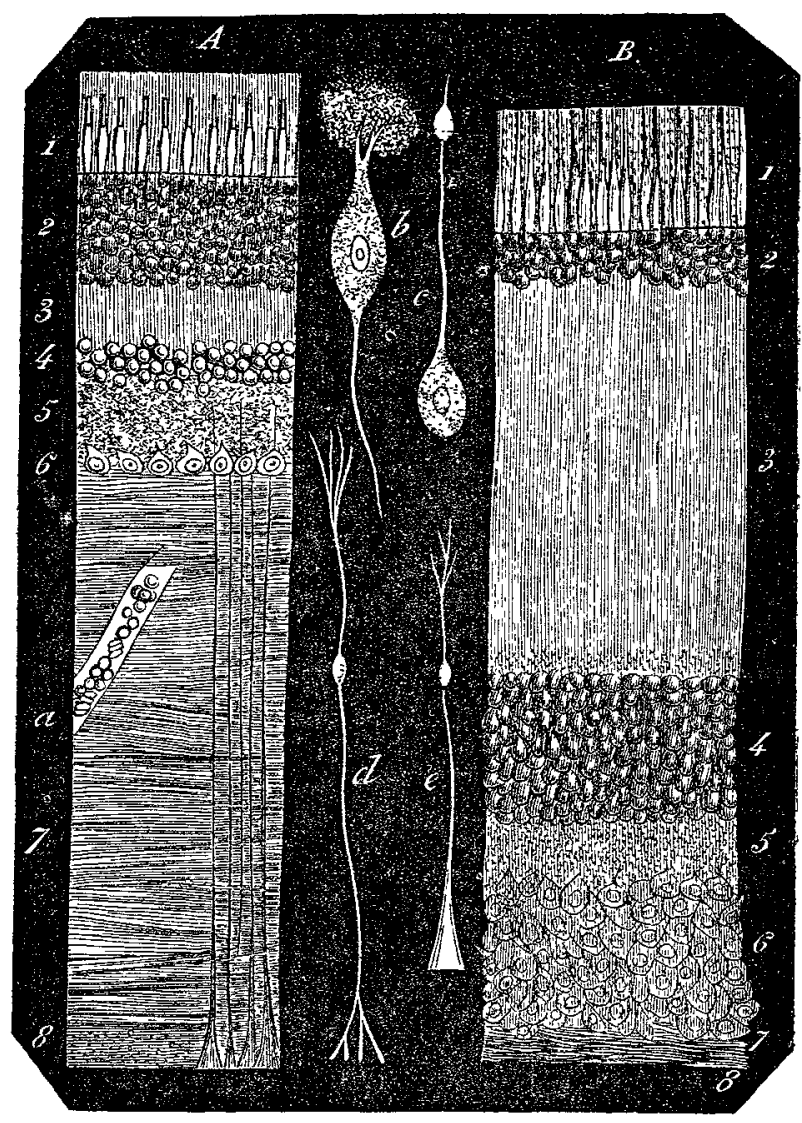

1. Layer of rods and coner.

2. External granular layer.

3. Intermediate layer.

4. La couche de myelocytes (fatty layer).

5. Grey granular layer.

6. Layer of nerve (or ganglion) cells,

7. Layer of nerve tubes.

But, with reference to the present investigation, the main point to be determined is, the part of the retina which is the immediate recipient of chromatic impressions. The view propounded by Young was, that there were three kinds of nervous fibres in the retina, each of which was specially endoweil with the power of receiving impressions from one colour only-that is, from red, green, or violet, -and that the varying intensity of impressions on these fibres produced, when combined together, the impressions of the other colours of the spectrum. This view has recently found an advocate in Helmholtz, who, while admitting that the selection of these colours is arbitrary, maintains that the hypothesis affords a satisfactory explanation of all the phenomena. of vision. Galezowski justly remarks that this rests on a pure assumption destitute of anatomical basis, and proposes the following explanation:-

It is admitted that the physical distinction between the various colours lies in the differing rapidity of their undulations and in their different refrangibility, and therefore the portion of the retina which is to perceive colours must be so arranged as to be capable of being impressed by both these properties. This double faculty, he thinks, is concentrated in the cones. A in Fig. 2 may represent the plan of a section of a cone, and $B$ the base of the same cone. The summit of each cone points to the interior of the eye, and from base to summit the cone is traversed by a central thread, which blends with the granular layer of the retina. A ray of light (s) which strikes the surface of the cone nearer the summit must, in traversing the cone, be refracted and decomposed, so as to produce at the base concentric circles corresponding to the solar spectrum, as shown at $\mathbf{B}$, where $a$ represents red, $b$ orange, $c$ yellow, $d$ green, $e$ blue, $f$ indigo, and $g$ violet. The concentric circles at the base will remain constantly sensible to the seven colours in such a manner that, if one colour only arrives-as, for example, blue,-it will make an impression only on the part blue (e) at the base, the others remaining without excitation. Supposing that white light arrives at the cone, it will be de- 
composed at the base; but as all the seven parts will be impressed, the result will be the impression of white light. The posterior layer of the retina, then-viz., the cones,receives the impressions of light and colour, and transmits them to the brain. By the mixture of these elementary colours nature furnishes us with an endless variety of colours and shades.

FIG. 2.

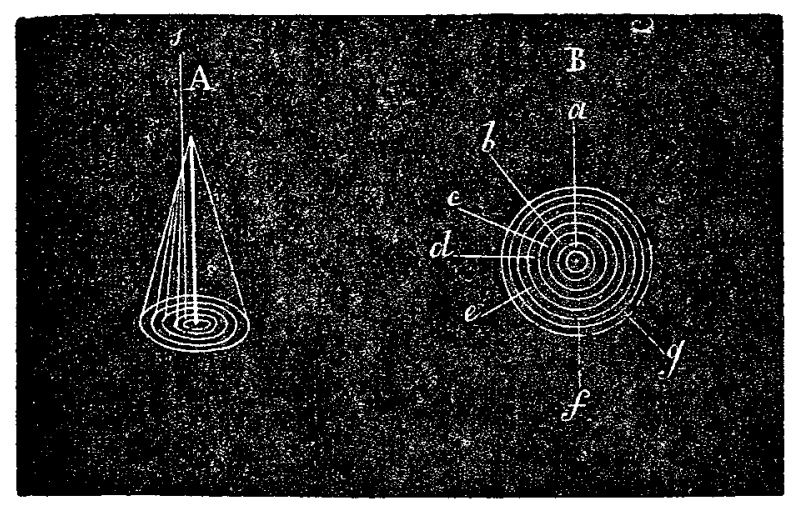

It is evident that all retinas are not capable of distinguishing so many shades, as not every ear can distinguish the different musical sounds. In addition to natural proneness to chromatic perception, it requires great practice and training to count 18,000 colours in the paintings of the Vatican but, on the other hand, it may be affirmed with an equal degree of certainty that a large proportion of those who habitually violate the law of contrast of colours in their dress and house decorations are affected with dyschromatopsy, and not simply with a vulgar or incongruous taste.

M. Chevreul has established 72 types of colours, of which he has constructed his chromatic tables. Galezowski selects eleven of the primary shades and four tints of each to form a standard chromatic scale for testing the colour-vision of patients. The colours selected are: red, orange-red, orange, orange-yellow, yellow, yellow-green, green, greenish-blue, blue, bluish-violet, and violet; that is, the primary colours with the middle shades between them. Each of these, again, is subdivided into four degrees of intensity, corresponding to the tints $1,5,10$, and 15 of Cherreul's scale. Thus a series of 44 colours is attained, all of which, according to M. Galezowski, ought to be recognisable by the healthy eye, and the confusion of any of which ought to be taken as an impairment of the colour sense of the eye.

Now, with regard to the pathology and its application to diagnosis we find-

I. Atrophy of the papilla, whether in its first or advanced stage, in addition to feebleness of vision, produces perversion of the chromatic faculty. This dischromatopsy is at first confined to secondary colours. The patient distinguishes well the principal colours, and even their shades, but they are embarrassed when they have to define the feeble tints. At a more advanced period of the disease the colour-blindness declares itself more prominently, especially in the case of the principal colours, red and green; the one appears grey, and the other black.

II. In alcoholic amblyopia, where the ophthalmoscopic examination gives a negative result, Galezowski's chromatic table is of great value; it reveals the phenomenon of pathological successive contrast of colours. We find a prolonged persistence of every coloured impression upon the retina; whence results a confusion of colours which vary constantly. Thus, then, patients recognise well every free, and even a composite, colour when they have had their eyes shut for some seconds; but as soon as they have fixed their eyes upon any colour-for instance, upon green-and then look upon red or orange, \&c.,一they maintain that they see the green more or less dark. Let them repose their eyes by shutting them for an instant, and we find immediately that they know well the red or orange; but during some time they still preserve the last impression.

III. In apoplexy of the retina the perversion of the chromatic faculty is not always decided; but where that phenomenon exists, it indicates alteration of the deep layers of the retina, particularly that of the rods and cones.

IV. Syphilitic choroiditis is generally accompanied with more or less disturbance of vision; the patient reads with difficulty No. 4. Where the retina had been affected at finst, they scarcely read No. 10 or 15 . The same phenomenon can be observed in syphilitic retino-choroiditis, either accompanied or not with turbidity of the vitreous; also in simple choroiditis, where the retina does not participate in the affection. It is in this last case that the diagnosis becomes difficult, especially when the vitreous is turbid, and the ophthalmoscope cannot discern retinal affections. Under these circumstances, it becomes absolutely impossible to $x$ ecognise whether the dimness of sight is owing only to the affection of the choroid and vitreous, or whether the visual nervous membrane is involved. Chromatoscopy elears ap this doubtful diagnosis: thus retinal change invariably produces a weakening of the chromatic faculty; whilst, when the vascular membrane alone is tainted by the virus, and the retina is unaffected, there is the conservation of that faculty intact. Syphilitic amblyopia is always accompanied with partial colour-blindness, especially of the compound colours, and of the less saturated shades which they compose. Thus green appears yellow or blue according as there is a predominancy of these colours in the green; violet appears red, and brown green, \&c. This is a valuable sign for all cases where a doubt may exist with regard to the probable alteration of the nervous membrane of the eve, which is very often, but not always, affected with syphilis, whilst the choroid is undamaged.

$\nabla$. In detachment of the retina, of which I have at present two cases under my care, I find that both patients can see red and green, but blue appears black, and yellow light or white colour. The necessity of researches of this kind eannot nowadays be called in question. It is easily understood that a diseased retina or a diseased optic nerve cannot discern colours and their shades so clearly as one which has undergone no structural changes. In comparing the numerous alterations of the internal membranes of the eye which the ophthalmoscope reveals, and in studying in each of these the degree of perversion or preservation of the chromatic faculty, we may succeed in drawing practical conclusions which may be utilised in the diagnosis of affections of the internal membranes of the eye. As stated at the outset of these remarks, we must not expect a well-defined system. There is no doubt something to add, to alter, and perhaps to abstract, as is the case with every new system. Further development, by the numerous distinguished labourers in that branch of science, may perhaps enable us to indicate with more precision the histological elements of the retina which perceives colours. With regard to the chromatic table I have only to notice that such attempts have already been made, but the great drawback has been that the colours do not stand the test of time and exposure, their tints gradually fading by the prolonged action of the air and light, so that the names become after a time inapplicable; but M. Galezowski's has the advantage that it can be renewed, as he has some printed separately from his work for the use of medical practitioners.

Glasgow, Jan. 1869.

ON A

\section{CASE OF POST-PARTUM HÆMORRHAGE}

Bx F. E. WILKINSON, M.D.

The following case may be of some interest as introducing a new mode of arresting uterine hæmorrhage. In the year 1860, I was called upon to attend Mrs. G-, an hæmorrhagic patient (a lady of the middle class), in her first confinement. Parturition was tedious, both from the length of time occupied, in consequence of the insufficiency of the uterine action, and from the occurrence of persistent vomiting; and delivery was accomplished with the aid of the forceps. The placenta was expelled by the natural efforts some twenty minutes after, but the uterus contracted feebly, and shortly afterwards severe hæmorrhage took place. I was here ably assisted by my son, Dr. G. F. E. Wilkinson, then a student of medicine, and with his aid ergot and food and stimulants were administered, and cold injections thrown up, but with no success; slight contraction taking place, but the uterus then becoming perfectly flaccid. Hæmorrhage continued, and the patient became rapidly de- 